\title{
Avaliação da Estabilidade Oxidativa do Biodiesel de Soja (Glycine max L.) na Presença de Antioxidantes Naturais Obtidos das Folhas de Acerola (Malpighia glabra L.) Utilizando $\mathrm{CO}_{2}$ Supercrítico. ${ }^{1}$
}

Ana Claudia dos Santos², Kátia Andressa Santos ${ }^{3}$, Edson da Silva ${ }^{4}$, Marcos Corazza ${ }^{5}$

${ }^{1}$ Aceito para publicação no $3^{\circ}$ trimestre de 2014

2 Mestranda em Bioenergia na Universidade Estadual do Oeste do Paraná- UNIOESTE, anasantos_cl@hotmail.com

3 Doutoranda em Engenharia Química na Universidade Estadual do Paraná- UNIOESTE. katiandressa@hotmail.com

${ }^{4}$ Professor Dr. do Departamento de Engenharia Química na Universidade Estadual do Oeste do Paraná- UNIOESTE, edsondeq@ hotmail.com

${ }^{5}$ Professor Dr. do Departamento de Engenharia Química na Universidade Federal do ParanáUFPR, corazza@ufpr.br.

\section{Resumo}

Biodiesel pode ser obtido de fontes renováveis, como óleos vegetais e devido ao seu perfil favorece a sua oxidação, sendo necessário acrescentar antioxidantes. Os mais utilizados são BHT, TBHQ e BHA. Os compostos fenólicos são importantes devido às suas propriedades antioxidantes. A aceroleira (Malpighia glabra L.) produz um fruto rico em vitamina $\mathrm{C}$ sendo cultivada em várias regiões do país. O objetivo desse trabalho foi avaliar a estabilidade oxidativa do Biodiesel na presença de antioxidantes sintético TBHQ e naturais dos extratos das folhas de Acerola obtidos com CO2 supercrítico em condições de temperatura e pressão variadas, utilizando Rancimat ${ }^{\circledR}$ para medição. Os melhores resultados foram para o Biodiesel acrescido de TBHQ. Os extratos nas condições $40{ }^{\circ} \mathrm{C}$ e 150 bar e $60{ }^{\circ} \mathrm{C}$ e 250 bar apresentaram tempo de indução maior que o controle.

Palavras-Chave: Antioxidantes naturais e sintéticos, Malpighia glabra L., Rancimat $^{\circledR}$. 


\title{
EVALUATION OF OXIDATIVE STABILITY OF BIODIESEL SOYBEAN (Glycine max L.) IN THE PRESENCE OF NATURAL ANTIOXIDANTS OBTAINED LEAVES ACEROLA (Malpighia glabra L.) USING CO2 SUPERCRITICAL
}

\begin{abstract}
Biodiesel can be obtained from renewable sources such as vegetable oils and due to their profile favors its oxidation, being necessary to add antioxidants. The most used are BHT, TBHQ and BHA. Phenolic compounds are important due to their antioxidant properties. Acerola (Malpighia glabra L.) produces a fruit rich in vitamin $\mathrm{C}$ being cultivated in many regions of the country. The aim of this study was to evaluate the oxidative stability of biodiesel in the presence of synthetic natural antioxidants TBHQ and Acerola extracts of leaves obtained with supercritical CO2 under conditions of varying temperature and pressure, using Rancimat ${ }^{\circledR}$ for measurement. The best results were for Biodiesel plus TBHQ. The extracts at $40^{\circ} \mathrm{C}$ and 150 bar and the conditions $60^{\circ} \mathrm{C}$ and 250 bar exhibited greater time than the control induction.
\end{abstract}

Keywords: Natural and synthetic antioxidants, Malpighia glabra L. and Rancimat ${ }^{\circledR}$.

\section{Introdução}

O Biodiesel não polui o ambiente e ainda apresenta vantagens econômicas, como geração de emprego. Pode ser obtido de fontes renováveis, como óleos vegetais, através da reação de transesterificação, que converte um triglicerídeo em um éster de ácido graxo. No Brasil a principal fonte de produção de biodiesel é a partir do Soja (Glicyne max L.). Os ácidos graxos presentes em óleos e gorduras, devido à quantidade de ácidos graxos insaturados, favorecem o processo de oxidação do Biodiesel. O Biodiesel é sensível à luz, pois, está sujeito a degradação por foto-oxidação. Este tipo de oxidação é um mecanismo que envolve a adição direta de um oxigênio singlete $\left({ }^{1} \mathrm{O}_{2}\right)$ aos ácidos graxos insaturados, esse oxigênio singlete reage com as duplas ligações presentes no óleo, produzindo hidroperóxidos, a forma mais importante de geração de oxigênio singlete é a exposição à luz na presença de um fotossensibilizador (Ferrari e Souza, 2009). O biodiesel, ainda, pode sofrer a autoxidação 
(oxidação causada pelo contato com o ar ambiente), o que acarreta na redução da sua qualidade durante o armazenamento.

Vários aditivos são adicionados à óleos com objetivo de inibir ou retardar reações indesejáveis (Lomonaco et al., 2011). Os tratamentos do biodiesel com antioxidantes são promissores, uma vez que facilitam a estocagem em estrutura já existentes. Os antioxidantes BHT e TBHQ são conhecidos por retardarem efeitos da oxidação na viscosidade, acidez e índice de peróxido no biodiesel (Duun, 2002).

A estabilidade oxidativa do biodiesel pode ser melhorada adicionando antioxidantes de ocorrência natural ou sintética (Focke et al., 2012), porém, antioxidantes de ocorrência natural mostraram serem menos eficientes quando comparados com os sintéticos na estabilidade à oxidação do biodiesel (Ball et al, 2010). Ao que se refere ao biodiesel, os antioxidantes podem retardar as reações de polimerização, porém, não inibi-las por completo (Graboski et al.,1998).

A Acerola (Malpighia glabra L.) é uma planta originária da América Central, foi introduzida no Brasil na década de 50, devido à sua boa adaptação ao solo e clima. Pertence à família Malpighiaceae (Asenjo, 1980 apud Mezadri et. al., 2008) e tem um pequeno fruto vermelho-cereja, sendo de grande importância nutricional por ser fonte natural de vitamina $\mathrm{C}$ e polifenóis que apresentam atividade antioxidante.

Compostos antioxidantes estão naturalmente presente em frutas, sendo que algumas apresentam altas concentrações de determinados grupos (Harborne et. al., 2000; Arabbi et. al., 2004; Duarte-Almeida et. al., 2006). A acerola apresenta grandes concentrações de ácido ascórbico (Assis et. al., 2001). Em extrato metanólico de resíduo de acerola (pele), foi evidenciado a presença de quantidade significante de compostos fenólicos e forte capacidade antioxidante (Oliveira et. al., 2009).

O objetivo geral de se extrair antioxidantes é liberar os compostos que apresentam tal atividade a fim de se obter extratos com alta atividade antioxidante. Desde os primórdios praticou-se a extração de substâncias através de matérias vegetais para fins alimentícios, aromáticos e de medicamentos. Dentre as várias técnicas utilizadas para extração o uso de fluídos supercríticos, em especial dióxido de carbono supercrítico $\left(\mathrm{CO}_{2}\right)$, é uma alternativa interessante devido as várias vantagens que apresenta (Ganán; Brignole, 2011). Além de que a utilização de $\mathrm{CO}_{2}$ supercrítico elimina a necessidade de evaporação do solvente do extrato obtido, onde o calor aplicado para tal fim acaba por degradar os compostos antioxidantes que 
são sensíveis ao calor. Uma desvantagem é que substâncias polares podem ser pouco solúveis em $\mathrm{CO}_{2}$ supercrítico (pouco polar) acarretando em um rendimento baixo de extração (Shi et al., 2011).

Assim, este trabalho visa avaliar a estabilidade oxidativa do Biodiesel na presença de antioxidantes naturais das folhas da Acerola (Malpighia glabra L.) obtidos com $\mathrm{CO}_{2}$ supercrítico, utilizando Rancimat $^{\circledR}$ para medição e analisando os efeitos das variáveis temperatura e pressão no rendimento e cinética da extração supercrítica realizada.

\section{Materiais e Métodos}

Preparo da Matéria-Prima

As folhas da Acerola foram coletadas na Região de Corbélia-Pr na época de Setembro de 2012. As folhas foram, inicialmente, armazenadas em sacos plásticos onde foram levadas ao laboratório para posteriormente serem desidratadas à secagem natural (Temperatura Ambiente) por um período de quatro dias. O material foi triturado e armazenado em sacos plásticos em refrigerador à $-15^{\circ} \mathrm{C}$.

O material triturado foi classificado de acordo com a série padrão de peneiras Tyler de 9 a 80 mesh. O material retido entre as peneiras de 12 e 48 mesh foram utilizadas nas extrações. Uma alíquota da matéria-prima foi levada para identificação no Herbário da Universidade Estadual do Oeste do Paraná (UNOP), a fim de confirmar a espécie e gênero da planta em estudo.

\section{Extração $\mathrm{CO}_{2}$ Supercrítico}

Os extratos foram obtidos a partir de uma unidade em escala laboratorial, sendo constituído de um reservatório para o solvente, uma bomba seringa, dois banhos termostáticos, onde, um é para resfriar o fluído na bomba e um para manter o extrator aquecido na temperatura necessária e um extrator de aço inox com capacidade de $80 \mathrm{~cm}^{3}$ (diâmetro de $2,52 \mathrm{~cm}$ e altura de $16 \mathrm{~cm}$ ).

Foi realizado um planejamento experimental $2^{2}$ com ponto central, a fim de se analisar a influência das variáveis independentes (temperatura e pressão) na obtenção de extrato das folhas de acerola. Os experimentos foram conduzidos nas temperaturas de 40, $50 \mathrm{e}$ $60{ }^{\circ} \mathrm{C}$, pressões de 150, 200 e 250 bar e vazão volumétrica de $2 \mathrm{~mL} \cdot \mathrm{min}^{-1}$. Os extratos foram coletados em intervalos de tempo pré-determinado durante 170 minutos. 


\section{Rendimento Globais da Extração Supercrítica}

Relaciona a quantidade de extrato final obtido pela quantidade de matéria-prima utilizada. Não foi contabilizado o extrato remanescente no extrator. $\mathrm{O}$ rendimento global pode ser calculado pela seguinte fórmula:

$$
R(\%)=\frac{m_{\text {extrato }}}{m_{\text {planta }}} \times 100
$$

Onde, $m_{\text {extrato }}$ é a massa total de extrato obtida e $m_{\text {planta }}$ é a massa de planta confinada no leito de extração.

\section{Modelagem Matemática}

Os modelos das isotermas de solubilidade são de fundamental importância na otimização e no projeto de extratores em escala industrial, O modelo de segunda ordem é usualmente empregado para representar a dinâmica de adsorção em colunas de leito fixo. $\mathrm{O}$ modelo empírico de segunda ordem foi adaptado para descrever a cinética de extração do óleo de candeia, e o modelo se mostrou bastante apropriado (SOUZA et al., 2008).

$\mathrm{O}$ modelo assume que a taxa de extração é proporcional à capacidade residual de extração do óleo pelo solvente $\left(\mathrm{C}_{\mathrm{eq}}-\mathrm{C}\right)$ e a concentração do óleo na matriz sólida. As taxas de transferência de massa obedecem às equações 2 e 3 :

$$
\begin{aligned}
& \frac{\partial C}{\partial t}+\frac{\rho_{b e d}}{\varepsilon} \frac{\partial q}{\partial t}+u \frac{\partial C}{\partial z}=0 \\
& \frac{\partial q}{\partial t}=-k q\left(C_{e q}-C\right)
\end{aligned}
$$

Onde:

$\mathrm{K}=$ constante cinética $\left(\mathrm{cm}^{3} \mathrm{~g} \mathrm{~min}^{-1}\right)$;

$\mathrm{C}_{\mathrm{eq}}=$ solubilidade do extrato no solvente $\left(\mathrm{g}_{\text {óleo }} \mathrm{cm}^{-3}\right)$

$\mathrm{u}=$ velocidade intersticial $\left(\mathrm{cm} \mathrm{min}^{-1}\right)$;

$\mathrm{q}=$ concentração do extrato na matriz sólida ( $\mathrm{g}$ óleo $\mathrm{g}$ sólido ${ }^{-1}$ );

$\mathrm{C}=$ concentração do extrato no solvente $\left(\mathrm{g} \mathrm{cm}^{-1}\right)$;

$\rho_{\text {bed }}=$ densidade do leito $\left(\mathrm{g} \mathrm{cm}^{-1}\right)$;

$\varepsilon=$ porosidade do leito;

$\mathrm{Z}=$ coordenada na direção axial do leito $(\mathrm{cm})$.

As condições inicias e de contorno são:

$$
C(0, z)=C_{e q}
$$




$$
\begin{aligned}
& q(0, z)=q_{0} \\
& C(t, 0)= \begin{cases}C_{e q} & t=0 \\
C_{0} & t>0\end{cases}
\end{aligned}
$$

A solução analítica deste modelo é dada pela equação (7):

$$
\frac{C_{\text {OUT }}}{C_{e q}}=\left\{\begin{array}{cl}
1 & t<t_{r} \\
\frac{1}{\left(e^{A}+e^{-b}-1\right) e^{B}} & t>t_{r}
\end{array}\right.
$$

Sendo: $\mathrm{t}_{\mathrm{r}} \mathrm{o}$ tempo de residência do $\mathrm{CO}_{2}$ no leito. As constantes são determinadas a seguir:

$$
\begin{aligned}
A & =\frac{z}{u} \beta \\
B & =\frac{(-t u+z) \beta}{\alpha u} \\
\beta & =k C_{e q} \alpha \\
\alpha & =\frac{\rho_{\text {bed }} q_{o}}{\varepsilon C_{e q}}
\end{aligned}
$$

O Parâmetro $k$ da taxa de extração pode ser estimado a partir dos dados experimentais. A massa de óleo extraída em função do tempo foi calculada pela equação (12):

$$
\begin{gathered}
m_{\text {óleo }}=\int_{0}^{t e} C_{\text {OUT }} w d t= \\
\begin{cases}C_{e q} w t & t<t_{r} \\
\left.\left.C_{e q} w t-\frac{C_{e q} w \alpha}{\beta} \ln \left(\exp \left(\frac{z \beta}{u}\right)+\exp (z-t v) \beta\right) / \alpha u\right)-1\right) & t>t_{r}\end{cases}
\end{gathered}
$$

Sendo: $w$ a vazão volumétrica do solvente e $C_{\text {OUT }}$ a concentração de óleo na fase fluída na saída da coluna.

O parâmetro $k$ foi calculado minimizando a função objetivo dada pela equação (13), usando os dados experimentais das curvas cinéticas.

$$
F_{O B J}=\sum_{J=1}^{n_{-} \exp }\left(m_{\text {oilJ }}^{\text {MOD }}-m_{\text {oilJ }}^{E X P}\right)^{2}
$$

Sendo: $m_{\text {oilj }}^{M O D}$ representa a massa de óleo que foi extraída pelo modelo, $m_{\text {oilJ }}^{E X P}$, é a massa de óleo extraída experimentalmente e $n \_\exp$ é o número de dados experimentais da curva cinética.

\section{Obtenção do Biodiesel}

Para obtenção do biodiesel o Óleo de Soja foi aquecido à $80{ }^{\circ} \mathrm{C}$ posteriormente adicionando uma solução de Metanol (30\% v/v) e Hidróxido de Potássio $(\mathrm{KOH})(1 \% \mathrm{~m} / \mathrm{m})$ com agitação contínua e aquecimento durante uma hora. A temperatura foi controlada à 60 
${ }^{\circ}$ C. Após, o produto obtido foi colocado em um balão de separação por vinte e quatro horas, decantando a fase da glicerina separando-a do biodiesel. Por fim, o biodiesel foi lavado com porções de água à $60^{\circ} \mathrm{C}$ para retirar impurezas e seco durante duas horas com temperatura constante de $60{ }^{\circ} \mathrm{C}$.

\section{Análise da Estabilidade do Biodiesel}

Foram preparadas amostras de $3 \mathrm{~g}$ de biodiesel de soja aditivado com os extratos naturais obtidos e TBHQ na proporção de 3000 ppm. Foram levados ao aquecimento acelerado a $110{ }^{\circ} \mathrm{C}$, com taxa de insuflação de ar de $10 \mathrm{~L} \mathrm{~h}^{-1}$, para determinação do período de indução. O teste foi realizado com o equipamento Rancimat 873 , em concordância com a Norma Oficial EN 14112 de determinação de estabilidade oxidativa em teste acelerado.

\section{Resultados e Discussão}

Rendimento Globais das Extrações

As condições experimentais de temperatura e pressão permitiram uma ampla variedade na densidade do dióxido de carbono supercrítico o que acarreta em uma ampla solvatação do mesmo. A tabela 1 apresenta as condições experimentais e os resultados dos rendimentos globais da extração supercrítica da Acerola.

Tabela 1: Condições experimentais para a extração supercrítica e rendimento global da extração

\begin{tabular}{llllll}
\hline Ensaio & $\begin{array}{l}\text { Temperatura } \\
\left({ }^{\mathbf{0}} \mathbf{C}\right)\end{array}$ & $\begin{array}{l}\text { Pressão } \\
(\mathbf{b a r})\end{array}$ & $\begin{array}{l}\text { Densidade do } \mathbf{C O}_{2} \\
\left(\mathbf{g ~ c m}^{-3}\right)\end{array}$ & $\begin{array}{l}\text { Tempo } \\
(\mathbf{m i n})\end{array}$ & $\begin{array}{l}\text { Rendimento } \\
(\%)\end{array}$ \\
\hline 1 & 40 & 150 & 0,781 & 170 & 0,47 \\
2 & 40 & 250 & 0,911 & 170 & 0,62 \\
3 & 60 & 150 & 0,607 & 170 & 0,41 \\
4 & 60 & 250 & 0,831 & 170 & 0,82 \\
5 & 50 & 200 & 0,785 & 170 & 0,60 \\
\hline
\end{tabular}

Os efeitos das variáveis são apresentados na Tabela 2, com coeficiente de determinação $\mathrm{R}^{2}$, de 0,99 . 
Tabela 2: Efeitos para as variáveis temperatura e pressão na obtenção do extrato das folhas de Acerola empregando $\mathrm{CO}_{2}$ supercrítico.

\begin{tabular}{lllll}
\hline Variável & Média/Intercepto & T & P & TxP \\
\hline Efeito & 0,587 & 0,070 & 0,280 & 0,130 \\
p-valor $^{\mathrm{a}}$ & 0,000 & 0,050 & 0,003 & 0,015 \\
Coeficiente & 0,587 & 0,035 & 0,140 & 0,065 \\
Erro padrão & 0,007 & 0,008 & 0,008 & 0,008 \\
\hline
\end{tabular}

${ }^{a}$ significância estatística p-valor $<0,05$

O maior rendimento foi de $0,82 \%$ obtido na condição de maior temperatura e pressão $\left(60{ }^{\circ} \mathrm{C}\right.$ e 250 bar). Para as condições utilizadas no planejamento fatorial as interações entre elas apresentaram efeito significativo sobre o rendimento da extração, sendo que a pressão exerce mais influência sobre a variável resposta.

Observa-se que o aumento da pressão em temperatura inalterada, acarreta no aumento do rendimento. O que pode ser explicado pelo aumento da densidade do solvente, o qual eleva o seu poder de solvatação. Outros autores também obtiveram o mesmo resultado (Campos et. al., 2008; Mantell et. al., 2002; Ribeiro et. al., 2007).

Ao que se refere a temperatura o efeito é mais complexo, já que em pressão mais baixa o rendimento diminui com o aumento da temperatura e, em pressão maior o contrário é observado (Almeida e Ferreira, 2007). Um aumento na temperatura acarreta no aumento da pressão de vapor do solvente, resultando um poder de solvatação maior, porém, com isso ocorre uma diminuição na densidade do $\mathrm{CO}_{2}$ consequentemente no poder de solvatação, como observado nas folhas de Acerola, onde a temperatura influiu mais na densidade do solvente que na pressão de vapor.

\section{Modelagem Matemática}

O valor da função objetivo é de grande importância já que indica o erro para cada modelo, assim, quanto menor o valor da função objetivo, menor o erro entre valores do modelo e os dados experimentais. Os valores de $k$, solubilidade e função objetivo estão presentes na Tabela 3. E como podemos observar os valores da função objetivo são baixos o que representa uma boa aproximação dos dados do modelo com os experimentais, sendo possível, assim, a determinação das curvas cinéticas das extrações experimentais e simuladas 
usando o modelo proposto. Não foi realizada a modelagem matemática para a condição $60{ }^{\circ} \mathrm{C}$ e 250 bar, por não se ajustar a curva cinética. As curvas cinéticas estão presentes na figura 2 .

Tabela 3: Parâmetros dos modelos cinéticos

\begin{tabular}{lllll}
\hline $\begin{array}{l}\text { Temperatura } \\
\left({ }^{\circ} \mathbf{C}\right)\end{array}$ & $\begin{array}{l}\text { Pressão } \\
(\mathbf{b a r})\end{array}$ & $\boldsymbol{k}$ & $\begin{array}{l}\text { Solubilidade } \\
\left(\mathbf{c m}^{\mathbf{2}} \mathbf{g} \mathbf{~ m i n}^{-\mathbf{1}}\right)\end{array}$ & \begin{tabular}{l} 
Função objetivo \\
\hline 40
\end{tabular} \\
\hline 150 & 37,81 & 0,000631 & 0,0005300 \\
40 & 250 & 15,00 & 0,001240 & 0,0000968 \\
50 & 200 & 23,88 & 0,000898 & 0,0005781 \\
60 & 150 & 136,04 & 0,000377 & 0,0005373 \\
\hline
\end{tabular}

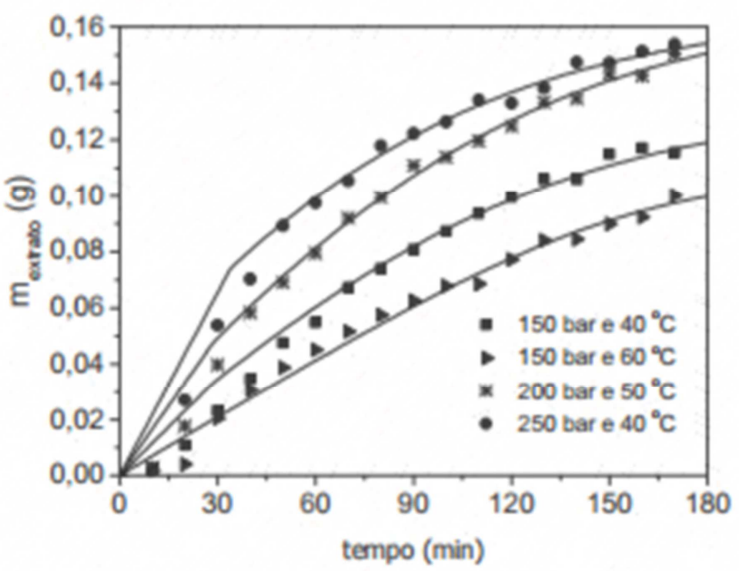

Figura 2: Ajuste cinético experimental para todas as condições empregadas

A cinética da extração varia com a solubilidade e o parâmetro $k$. Pela análise das curvas observa-se que a massa de óleo extraída nos tempos iniciais foram muito próximas para as condições $40{ }^{\circ} \mathrm{C}$ e 150 bar e $60{ }^{\circ} \mathrm{C}$ e 150 bar, porém, a cinética da condição $40{ }^{\circ} \mathrm{C}$ e 150 bar foi mais rápida devido a maior solubilidade do óleo.

A condição $40^{\circ} \mathrm{C}$ e 250 bar foi a mais rápida, ao comparar-se as quatro condições, devido ao seu maior valor de solubilidade. Assim, pode-se afirmar que a velocidade da extração aumenta com o aumento da solubilidade.

\section{Análise da Estabilidade do Biodiesel}

Para avaliar a estabilidade oxidativa ou a susceptibilidade à oxidação, o biodiesel (B100), acrescidos de antioxidantes foi submetido ao teste de oxidação acelerada. A Revista Brasileira de Energias Renováveis, v. 3, p. 197- 210, 2014 
especificação estabelecida pela Norma EN 14112 indica que o período de indução mínimo deve ser de 6 horas, à $110^{\circ} \mathrm{C}$. De acordo com os valores para o tempo de indução obtidos, presentes na Tabela 4 , pode-se perceber que apenas o biodiesel na presença de antioxidante sintético (TBHQ) obteve um tempo de indução satisfatório. Para os demais o tempo de indução foi inferior à 6 horas, porém, para o biodiesel acrescidos dos antioxidantes naturais presentes no extrato das folhas de Acerola obtido com $\mathrm{CO}_{2}$ supercrítico nas condições $40{ }^{\circ} \mathrm{C}$ e 150 bar e $60{ }^{\circ} \mathrm{C}$ e 250 bar o tempo de indução foi superior ao tempo de indução do Controle (Biodiesel puro, sem antioxidantes) demonstrando uma possível ação antioxidante dos extratos, no entanto, não suficiente para elevar o período de indução a um valor superior às 6 horas determinadas pela ANP.

Tabela 4: Tempo de indução para Biodiesel de Soja na presença de antioxidantes naturais e sintético

\begin{tabular}{ll}
\hline Condição & $\begin{array}{l}\text { Tempo de indução } \\
\text { (horas) }\end{array}$ \\
\hline Controle 1 & 1,04 \\
Controle 2 & 0,91 \\
TBHQ & 36,95 \\
Extrato $40^{\circ} \mathrm{C}$ e 150 bar & 1,38 \\
Extrato $40^{\circ} \mathrm{C}$ e 250 bar & 0,90 \\
Extrato $50^{\circ} \mathrm{C}$ e 200 bar & 0,17 \\
Extrato $60^{\circ} \mathrm{C}$ e 150 bar & 0,77 \\
Extrato $60^{\circ} \mathrm{C}$ e 250 bar & 1,07 \\
\hline
\end{tabular}

Muitos estudos têm mostrado a eficiência do TBHQ frente a outros antioxidantes existentes. Ferrari e Souza (2008), avaliaram a estabilidade oxidativa de biodiesel de óleo de girassol na presença de antioxidantes sintéticos (BHA, BHT e TBHQ) na proporção de 0,5\%, obtendo tempos de indução inferiores à 6 horas, exceto para o TBHQ que o tempo foi superior (6h30min), o controle para esse trabalho obteve tempo de indução de $1 \mathrm{~h} 10 \mathrm{~min}$.

Mittelbach e Schober (2003) estudaram a influência de antioxidantes naturais e sintéticos na estabilidade oxidativa do biodiesel, utilizando teste Rancimat ${ }^{\circledR}$ para medição. Foram analisados biodiesel de canola, girassol, fritura e sebo animal, acrescidos de 100 ppm 
dos antioxidantes pirogalol (PY), propilgalato (PG), TBHQ, BHT e BHA. Os melhores valores obtidos foram para PY, PG e TBHQ.

O efeito da concentração do antioxidante sobre a taxa de auto-oxidação do biodiesel depende de muitos fatores, dentre os quais, estrutura do antioxidante, condições de armazenamento, bem como, a natureza/composição da amostra a ser oxidada (Fattah et al., 2014). Como exemplo, Mittelbach e Schober (2003) obtiveram a menor estabilidade oxidativa para o Biodiesel obtido de óleo de girassol em comparação com os demais, isto deve-se ao fato desse óleo apresentar maior concentração de ácido linoléico, que é menos estável à oxidação que o ácido oleico.

Luo et al., (2012) analisaram a estabilidade do biodiesel de soja na presença de cinco aditivos naturais como antioxidantes (ácido felúrico (FA), polifenol de chá lipídico-solúvel (LTP), polifenol de chá solúvel em água (WTP), sesamol (SE) e ácido cafeico (CA)), os resultados obtidos para os antioxidantes naturais WTP, LTP, SE e CA foram melhores para os antioxidantes sintéticos ( $\alpha$-TBHQ e BHA), uma possível explicação seria a estrutura molecular dos antioxidantes.

\section{Conclusões}

Analisando os resultados pode-se confirmar a possibilidade do uso das folhas de acerola para obtenção de extrato através do $\mathrm{CO}_{2}$ supercrítico. O maior rendimento obtido de 0,82\% na condição de maior temperatura e pressão e comparando com os demais valores pode-se observar uma maior influência da pressão no rendimento das extrações. O modelo de segunda ordem representou satisfatoriamente os dados experimentais em todas as condições empregadas. O biodiesel teve um maior tempo de indução na presença do antioxidante sintético TBHQ. Os extratos das condições $40{ }^{\circ} \mathrm{C}$ e 150 bar apresentou o melhor tempo de indução dos antioxidantes naturais, sendo superior ao controle, porém, ainda inferior às 6 horas determinadas pela ANP.

\section{Referências}

ALMEIDA, P. P.; FERREIRA, S. R. S. Crossover pressure for supercritical fluid extraction of spearmint (Mentha spicat L, ) essential oil with pure $\mathrm{CO}_{2}$ and $\mathrm{CO}_{2}$ plus ethanol. In: I Iberoamerican Conference on Supercritical Fluids (PROSCIBA), Foz do Iguaçu, Paraná, Caderno de Resumos do PROSCIBA, 2007. 
ARABBI, P.R.; GENOVESE, M.I.; LAJOLO, F.M. Flavonoids in vegetable foods commuly consumed in Brazil end estimated by the brazilian population. Journal of Agricultural and Food Chemistry, v. 52, p. 1.124-1.131, 2004.

ASSIS, S.A.; LIMA, D.C.; OLIVEIRA, O.M.M.F. Activity of pectinmethylesterase, pectin content and vitamin $C$ in acerola fruit at various stages of fruit development. Food Chemistry, v. 74, p. 133-137, 2001.

BAll, J., SHAH, R., MAHAJAN, D., PATEL, S., COLANTUONI, V., MARAJ, R.; Oxidation stability in biodiesel: a brief review of current technology. $<$ (http://www.biodieselmagazine.com/article-print.jsp?article_id=3541>; 2009. Acesso 03.09.14.

CAMPOS, L.M.A.S., LEIMANN, F.V., PEDROSA, R.C., FERREIRA, S.R.S., Free radical scavenging of grape pomace extracts from Cabernet sauvingnon (Vitis vinifera). Bioresource Technology, 2008.

DUARTE-AlMEIDA, J. M.; SANTOS, R. J. dos.; GENOVESE, M. I.; LAJOLO, F. M.; Avaliação da atividade antioxidante utilizando sistema $\beta$-caroteno/Ácido linoleico e método de sequestro de radicais DPPH. Ciência e Tecnologia de Alimentos, Campinas, 26(2): 446452, abr.-jun. 2006.

DUNN, R. O.; Effect of oxidation under accelerated conditions on fuel properties of methyl soyate (biodiesel). Journal of the American Oil Chemists' Society, Vol. 79, 915-920, 2002.

FATTAH, I. M. R.; MASJUKI, H. H.; KALAM, M. A.; HAZRAT, M. A.; MASUM, B. M.; IMTENAN, S.; ASHRAFUL, A. M.; Effect of antioxidants on oxidation stability of biodiesel derived from vegetable and animal based feedstocks. Renewable and Sustainable Energy Reviews. Vol. 30, 356-370, 2014.

FERRARI, A. R.; SOUZA, W. L. de.; Avaliação da estabilidade oxidativa de biodiesel de óleo de girassol com antioxidantes. Química Nova, Vol. 32, No. 1, 106-111, 2009. 
FOCKE, W. W.; WESTHUIZEN, I. V. der.; GROBLER, A. B. L.; NSHOANE, K. T.; REDDY, J. K.; LUYT, A. S.; The effect of synthetic antioxidants on the oxidative stability of biodiesel. Fuel. Vol. 94, 227-233, 2012.

GANAN, N.; BRIGNOLE, E. A.; VELEZ, A.; HEGEL, P.; Semi-continuous fractionation of bioactive essential oils with supercritical carbon dioxide. Experiments, modeling and separation of stereoisomers. Disponível em http://www.isasf.net/fileadmin/files/Docs/DenHaag/HtmlDir/Papers/P34.pdf> Acesso 03/09/2014.

GRABOSKI, MS., MCCORMICK, R. L.; Combustion of fat and vegetable oil derived fuels. In diesel engines. Progress in Energy and Combustion Science. Vol. 24, 126-128, 1998.

HARBORNE, J.B.; WILLIANS, C.A. Advances in flavonoid research since 992. Phytochemistry, v. 55, n. 6, p. 481-504. 2000.

LOMANOCO, D.; MAIA, F. J. N.; CLEMENTE, C. S.; MOTA, J. P. F.; COSTA Jr, A. E.; MAZZETTO, S. E.; Thermal studies of new biodiesel antioxidants synthesized from a natural occurring phenolic lipid. Fuel. Vol. 97, 552-559, 2011.

LUO, M.; ZHANG, R.; ZHENG, Z.; WANG, J.; JI, J.; Impact of Some Natural Derivatives on the Oxidative Stability of Soybean Oil Based Biodiesel. Journal of the Brazilian Chemical Society, Vol. 23, No. 2, 241-246, 2012.

MANTELL, C.; RODRÍGUES, M.; MARTÍNEZ DE LA OSSA, E. Semi-Batch extraction of anthocyanins form red grape pomace in packed beds: experimental results and process modeling. Chemical Engineering Science, Vol. 57, 3831-3838, 2002.

MEZADRI, T.; VILLAÑO, D.; FERNÁNDEZ-PACHÓN, M. S.; GARCÍA-PARRILLA, M. C.; TRONCOSO, A. M.; Antioxidant compounds and antioxidant activity in acerola (Malpighia emarginata DC.) fruits and derivatives. Journal of Food Composition and Analysis. Vol. 21, 282- 290, 2008.

Revista Brasileira de Energias Renováveis, v. 3, p. 197- 210, 2014 
MITTELBACH, M,; SCHOBER, S.; The Influence of Antioxidants on the Oxidation Stability of Biodiesel. Journal of the American Oil Chemists' Society, Vol. 80, No. 8, 2003.

OLIVEIRA, A.C.;VALENTIM, I.B.; SILVA, C.A., HENRIQUES BECHARA, E.J.; DE BARROS, M.P.; MANO, C.M.; FONSECA GOULART, L. O.; Total phenolic content and free radical scavenging activities of methanolic extract powders of tropical fruit residues. Food Chemistry, London. Vol. 115. No 2. 469-475. 2009.

RIBEIRO, É. T. S.; FETT, R.; FERREIRA, S.R. S. Supercritical fluid extraction of apple pomace: phenolics compounds and in vitro antioxidant activity. In: I Iberoamerican Conference on Supercritical Fluids (PROSCIBA), Foz do Iguaçu, Paraná. Caderno de Resumos do PROSCIBA, 2007.

SHI, L.-E.; ZHANG, Z.-L.; XING, L.-Y.; YANG, D.-D.; GUO, Y.-P.; GUO, X.-F.; ZHAO, L.-M.; TANG, Z.-X. Antioxidants extraction by supercritical CO2. Journal of Medicinal Plants Research, v. 5, n. 3, p. 300-308, 2011.

SOUZA, A. T.; BENAZZI, T. L.; GRINGS, M. B.; CABRAL, V.; SILVA, E. A.; CARDOZO FILHO, L.; ANTUNES, O. A. C. Supercritical extraction process and phase equilibrium of Candeia (Eremanthus erithropappus) oil using 92 supercritical carbon dioxide. The Journal of Supercritical Fluids. v. 47, p. 182-187, 2008. 